Editorial

\title{
Special Issue on Immune Responses in Tumors and Non-Transformed Inflammatory Microenvironments
}

\author{
Anahid Jewett ${ }^{凶}$ \\ Division of Oral Biology and Medicine, Jonsson Comprehensive Cancer Center, UCLA School of Dentistry, Los Angeles, CA, USA \\ $\triangle$ Corresponding author: Email: ajewett@ucla.edu \\ () Ivyspring International Publisher. This is an open-access article distributed under the terms of the Creative Commons License (http://creativecommons.org/ \\ licenses/by-nc-nd/3.0/). Reproduction is permitted for personal, noncommercial use, provided that the article is in whole, unmodified, and properly cited.
}

Published: 2012.11 .30

Recent advances in our understanding of immune responses in tumors as well as in non-transformed inflammatory microenvironments have revealed a complex dynamic interaction between the immune effectors, other healthy stromal cells and the tumor cells in inhibition or progression of tumors and in the regulation of inflammation. The function of immune system in fight against bacterial and viral infections, and malignancies have clearly been highlighted and studied in many important previous studies; however, recent data from several laboratories have identified unexpected novel dimension to the function of immune cells. This includes selection and differentiation of cancer stem cells and in the regulation of inflammation for the ultimate goal of tissue regeneration and repair. Although, the former function of immune cells have received significant attention by the immunologists and cancer biologists, it is only recently that many investigators have turned their attention to the second important function of immune cells, which deals with the regulation of inflammation for the ultimate goal of tissue regeneration, and for the maintenance of the normal structural and functional integrity of the tissues to prevent carcinogenesis and other pathological alterations.

Tumor microenvironment is known to be immunosuppressive, and a great deal of effort has gone to reverse such immunosuppression in cancer patients. Unfortunately, despite many innovative and ground breaking strategies to reverse immunosuppression, disappointing results have been obtained in cancer patients thus far. Therefore, although paradoxical in nature, immunosuppression in tumors ap- pears to be an important functional attempt of the immune effectors for the repair and regeneration of the tissues, and more importantly for the resolution of inflammation, which is known to fuel tumor growth. Thus, significant future efforts may be concentrated in finding whether modulating regulatory cell functions in tumors is friend or foe. This collection of reviews highlights the complexity of the function of immune cells in the tumor - as well as in non-transformed inflammatory microenvironments emphasizing the significance of key subsets of immune effectors and their secreted cytokines, and newly characterized regulatory cells in immune suppression in tumors, and their potential in promotion or inhibition of tumorigenesis.

Drs. Sevko and Umansky [1] provide strategies in their review that may improve treatment outcomes in cancer patients by targeting and removing the Myeloid Derived Suppressor Cells (MDSCs), which are known to provide immunosuppression in tumor microenvironment. They provide a comprehensive review of the function, generation and control of MDSCs in tumor microenvironment. The relationship between the tumors and MDSCs is discussed in depth.

In their review [2], Dr. Jewett and colleagues discuss the dual function of human NK cells in selection and differentiation of cancer stem cells and their role in regulation of inflammation. The concept of split anergy in NK cells and induction of regulatory NK cells $\left(\mathrm{NK}_{\mathrm{reg}}\right)$ and its contribution to cell differentiation, tissue repair and regeneration and in tumor resistance is discussed.

In their review [3], Drs. Palmer and Malarkan- 
nan discuss the duality of NK cell function in stimulation of the immune system, and also in the regulation of immune responses to control the immune system. The duality of the function of NK cells is critical to understand, in particular when considering therapeutic interventions against tumors. Such regulatory functions by the NK cells may be the key not only in controlling graft versus host disease (GvHD) but also in the proper functioning of mucosal epithelial cells.

In their review [4], Drs. Ma and Shurin discuss the different subsets and functions of DCs and propose that better understanding of DC immunobiology in cancer is pivotal for designing novel or improved therapeutic approaches that will allow proper functioning of DCs in patients with cancer. DCs represent special subsets of immune effectors which display different phenotype and activity at the tumor site and exhibit differential pro-tumorigenic and anti-tumorigenic functions. DCs play a key role in inducing and maintaining the antitumor immunity, but in the tumor environment their antigen-presenting function may be lost or inefficient. DCs might also be polarized into immunosuppressive/tolerogenic regulatory DCs, which limit activity of effector $\mathrm{T}$ cells and may support tumor growth and progression.

The review [5] by Drs. Magister and Kos on the role of cystatins and cathepsins in the regulation of dendritic cell (DC) maturation and NK cell functional inactivation highlights important upstream regulators of immune effector function which may enhance tumor survival. They discuss the current knowledge on type I (stefins A and B) and type II cystatins (cystatins $\mathrm{C}, \mathrm{F}$ and $\mathrm{E} / \mathrm{M}$ ) in disease pathologies, with particular emphasis on their suppressive vs. promotional effect in tumorigenesis and metastasis. They propose that an imbalance between cathepsins and cystatins may attenuate immune cell functions and facilitate tumor cell invasion.

In their review [6] Drs. Kumar and Malarkannan indicate that defined immune paradigm involves recognition and elimination of virus-infected epithelial cells. However, they also acknowledge that evidence is mounting regarding the involvement of immune cells in regulation and regeneration of destroyed tissues. They present ample evidence for the role of IL-22 produced by key immune effectors in regeneration of epithelial cells. They propose that the future studies should focus on the role of the immune system in the maintenance and differentiation of stem cells by immune cells and their mediators.

In his review [7] Dr. Koontongkaew highlights the significance of tumor cell and cancer associated fibroblast interaction in tumor microenvironment in invasion and metastasis of Head and Neck Squamous
Cell Carcinomas. For tumor cells that survive in the tumor microenvironment, he suggests that stromal activation may serve to establish a supportive tumor stroma, fostering the outgrowth of the metastatic cells. Therefore, understanding the complex interplay between the effectors of the stroma with the tumor cells may be crucial for designing strategies for diagnosis, prognosis and therapy of HNSCC.

Dr. Man and colleague [8] discuss the existing proposed hypotheses and models that favor the role of tumor-infiltrating immune cells in enhancing tumor invasion and metastasis, and analyze their strengths and weaknesses. They also provide discussions on potential tumor lymphocyte chimeras (TLC) which may facilitate the transport and invasion of tumors, and may also represent a morphologically defined precursor for metastatic lesions of most epithelium-derived cancers.

The corresponding authors of these review articles are well-established and regarded investigators, with an average of about 100 publications each in the field. The main contents presented in their article(s) are largely based on the most recent studies of their own. Collectively, these articles have summarized the past, analyzed the current, and elucidated the future efforts and directions to facilitate our understanding of immune responses in tumors as well as in non-transformed inflammatory microenvironments.

\section{References}

1. Sevko A, Umansky V. Myeloid-Derived Suppressor Cells Interact with Tumors in Terms of Myelopoiesis, Tumorigenesis and Immunosuppression: Thick as Thieves. J Cancer 2013; 4(1):3-11.

2. Jewett A, Man YG, Tseng HC. Dual Functions of Natural Killer Cells in Selection and Differentiation of Stem Cells; Role in Regulation of Inflammation and Regeneration of Tissues. J Cancer 2013; 4(1):12-24.

3. Palmer JM, Rajasekaran K, Thakar MS, Malarkannan S. Clinical Relevance of Natural Killer Cells Following Hematopoietic Stem Cell Transplantation. J Cancer 2013; 4(1):25-35.

4. Ma Y, Shurin GV, Peiyuan Z, Shurin MR. Dendritic Cells in the Cancer Microenvironment. J Cancer 2013; 4(1):36-44.

5. Magister Š, Kos J. Cystatins in Immune System. J Cancer 2013; 4(1):45-56.

6. Kumar P, Rajasekaran K, Palmer JM, Thakar MS, Malarkannan S. IL-22: An Evolutionary Missing-Link Authenticating the Role of the Immune System in Tissue Regeneration. J Cancer 2013; 4(1):57-65.

7. Koontongkaew S. The Tumor Microenvironment Contribution to Development, Growth, Invasion and Metastasis of Head and Neck Squamous Cell Carcinomas. J Cancer 2013; 4(1):66-83.

8. Man Yg, Stojadinovic A, Mason J, Avital I, Bilchik A, Bruecher B, Protic M, Nissan A, Izadjoo M, Zhang X, Jewett A. Tumor-Infiltrating Immune Cells Promoting Tumor Invasion and Metastasis: Existing Theories. J Cancer 2013; 4(1):84-95. 